\title{
El proceso de vacunación en México.
}

\author{
Mexico's vaccination process.
}

\author{
José Eduardo Orellana Centeno,* Roxana Nayeli Guerrero Sotelo ${ }^{\ddagger}$
}

\section{RESUMEN}

La vacunación es un proceso de importancia para todos los países del mundo como una medida preventiva para la enfermedad del virus SARS-CoV-2. El proceso de adquisición, distribución, prioridad de grupos de la población a vacunar y la forma de acceder a ellas depende cada país. México en tasa bruta es el tercer país del mundo con mayor cantidad de muertos y la importancia de tener vacuna y medicamentos para tener un tratamiento es vital para disminuir la morbilidad y mortalidad a nivel mundial. El propósito del presente ensayo es mostrar el proceso de distribución y desarrollo de la vacunación en México.

Palabras clave: Vacunación, México, coronavirus, prevención, salud pública.

\section{ABSTRACT}

Vaccination is an important process for all countries in the world as a preventive measure for the SARS-CoV-2 virus disease, the process of acquisition, distribution, priority of population groups to be vaccinated and the way to access them depends on each country. Mexico in gross rate is the third country in the world with the highest number of deaths and the importance of having vaccine and drugs for treatment is vital to reduce morbidity and mortality worldwide. The purpose of this essay is to present the process of distribution and development of vaccination in Mexico.

Keywords: Vaccination, Mexico, coronavirus, prevention, public health.

\section{Qué se sabe sobre el tema}

L a pandemia de COVID-19 (coronavirus) es uno de ـ los temas de actualidad en salud pública debido a lo rápido que ha fluido la información, desarrollado prácticamente en un año, y el enfoque a estudiar lo más que se pueda sobre el tema desde la generalidad, pero de las particularidades y formas de atender a la población en cada uno de los distintos países. El proceso de vacunación ha sido lento a pesar de que el desarrollo en la producción de vacunas ha sido rápido, con una gran variedad de ellas producidas por distintos laboratorios. La distribución de la vacunación va desde ser de manera masiva en países como Estados Unidos hasta en grupos etarios como sucede en México.

\section{Qué se aporta en este trabajo}

La vacunación en México tiene un proceso particular, el cual quedó implementado a finales del año 2020, pero conforme fueron llegando las vacunas ha sufrido algunas modificaciones, considerando para su distribución, en primer lugar, a grupos etarios específicos; después, a las vacunas y medicamentos que existen hasta el momento para atender a la población y, finalmente, a cuestiones políticas involucradas en la distribución y aplicación. Todo lo anterior tuvo como eje central al mismo presidente de la República y a un grupo desarrollado para realizar las aplicaciones de las vacunas.

En México, se han rebasado las 150,000 muertes y parece que no existe la manera de detener los contagios

\footnotetext{
* Doctorante en Educación. Profesor Investigador de tiempo completo de la Licenciatura en Odontología.

₹ Doctora en Estudios Sociales. Profesor Investigador de tiempo completo de la Licenciatura en Enfermería.

Instituto de Investigación Sobre la Salud Pública. Universidad de la Sierra Sur. Oaxaca, Miahuatlán de Porfirio Díaz, México.

Recibido: 26 de septiembre de 2021. Aceptado: 04 de octubre de 2021.

Citar como: Orellana CJE, Guerrero SRN. El proceso de vacunación en México. Rev ADM. 2021; 78 (5): 270-274. https://dx.doi.org/10.35366/102034
}

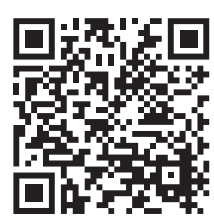


y las muertes. Existe un exceso de muertes entre enero y agosto del 2020 con 683,823 por parte del INEGI con respecto a los 499,784 reportados por la Secretaría de Salud (diferencia de 184,039).

\section{VACUNAS}

El desarrollo de una vacuna eficaz es un proceso complejo que requiere una gran cantidad de recursos (humanos, económicos, etc.) y de infraestructura tecnológica (industria, laboratorios, equipos e instrumentos), así como también requiere mucho tiempo entre los procesos de producción y distintas fases de experimentación (nivel básico de laboratorio y ensayos clínico). Siendo todo un desafío para desarrollar vacunas anti-COVID-19, logrando eficacia y seguridad clínica. ${ }^{1}$

Las vacunas son un elemento importante para que disminuya el número de casos y la mortalidad provocada por el virus SARS-CoV-2 en la población; ésta es una de las razones por las que a nivel mundial se desarrolló de manera acelerada la producción y desarrollo de vacunas en periodos de menos de un año. ${ }^{2}$

Hasta el 12 de noviembre del 2020 están registradas en la plataforma de vacunas candidatas, ante la OMS, 164 vacunas y 48 de ellas se encuentran en proceso de fases clínicas. Las vacunas desarrolladas hasta ese momento tienen diferente componente como:
1. Basado en ácido ribonucleico (RNA)

2. Basado en ácido desoxirribonucleico (DNA)

3. Basado en subunidades de proteínas recombinantes

4. Virus inactivado

5. Vector viral no replicado

6. Virus vivo atenuado

7. Vector de replicación bacteriana

8. Basado en células $T^{3}$

Hasta el momento, las que tienen una aprobación de emergencia por la Agencia de Alimentos y Medicamentos (FDA) y la Agencia Europea de Medicamentos (EMA) son la vacuna de Moderna (mRNA-1273), Pfizer \& BioNTech (BNT162) y la de AstraZeneca y la Universidad de Oxford (AZD1222) (Tabla 1). ${ }^{4}$

\section{MEDICAMENTOS}

En la actualidad, no existe un protocolo o manejo para el tratamiento de la enfermedad COVID-19, solamente se cuenta con las estrategias establecidas por los distintos gobiernos, como son la buena higiene y distanciamiento social para reducir la transmisión del virus. ${ }^{5}$

Dentro de los medicamentos propuestos se encuentran antivirales y antipalúdicos como tratamientos dirigidos para limitar los efectos adversos del virus.

\begin{tabular}{rccc}
\multicolumn{2}{c}{ Tabla 1: Vacunación mundial (27 de enero del 2021). } \\
& País & $\begin{array}{c}\text { Número de personas vacunadas } \\
\text { (millones) }\end{array}$ & \\
& Israel & 50.22 & Vacuna \\
\hline 1 & Emiratos Árabes Unidos & 29.00 & Pfizer \& BioNTech \\
2 & Reino Unido & 11.67 & Sputnik V \\
3 & Estados Unidos de América & 7.45 & Pfizer \& BioNTech \\
4 & & & Pfizer \& BioNTech \\
& Malta & 5.33 & Moderna \\
5 & Serbia & 5.32 & Pfizer \& BioNTech \\
6 & & & Sputnik V \\
& & Sinopharm \\
7 & Islandia & 4.55 & Pfizer \& BioNTech \\
8 & Dinamarca & 3.82 & Pfizer \& BioNTech \\
9 & Rumania & 3.17 & Pfizer \& BioNTech \\
10 & Portugal & 2.90 & Pfizer \& BioNTech \\
29 & Argentina & SpioNTech \\
31 & México & 0.73 & Sputnik V \\
& & 0.51 & Pfizer \& BioNTech \\
\hline
\end{tabular}

Fuente: Our World in Data.

\footnotetext{
i Gobierno de México. Exceso de mortalidad en México. Disponible en: https://coronavirus.gob.mx/exceso-de-mortalidad-en-mexico/
} 
Tabla 2: Medicamentos candidatos.

\begin{tabular}{lc} 
Medicamento & Fase clínica \\
\hline Remdesivir & II/III \\
Favipiravir & III \\
Ribavirin & II \\
Oseltamivir & III \\
Galidesivir & II \\
Sofosbuvir & II/III \\
EIDD-2801 & II \\
Lopinavir/ritonavir & II \\
Ivermectina & III \\
Arbidol & III \\
Darunavir & III \\
Clonidina & II \\
Brensocatib & III \\
Truvada & III \\
Hidroxicloroquina/cloroquina & III \\
\hline Modificada de: Mishra SK et al. &
\end{tabular}

\section{LOS ANTIVIRALES}

1. Atacan proteínas específicas interrumpiendo etapas de crecimiento en el ciclo vital del virus.

2. Contra el RNA común.

3. Inhibidores de la transcriptasa inversa.

4. Inhibidores de RdRp.

5. Inhibidores de proteasa (TMPRSS2 y otras proteasas que están envueltas en el procesamiento del polipéptido de cadena larga codificado por ORF1) (Tabla 2). ${ }^{6}$

La Organización Mundial de la Salud conduce un estudio multicéntrico global llamado Ensayo Clínico Solidaridad (Solidarity Plus Trial). Este ensayo reclutó cerca de 12 mil pacientes en 500 hospitales ubicados en 30 países, para investigar el potencial de cuatro medicamentos utilizados como son: remdesivir, hidroxicloroquina, lopinavir/ ritonavir e interferón- $\beta 1$ a. Dicho ensayo demostró que los medicamentos mencionados tienen bajo o nulo efecto en la disminución de mortalidad de pacientes hospitalizados por COVID-19. ${ }^{\text {? }}$

\section{PROCESO DE VACUNACIÓN EN MÉXICO}

Se desarrolló una estrategia de vacunación plasmada en el documento que se conoce como Política Nacio- nal de Vacunación Contra el Virus SARS-CoV-2 para la prevención de la COVID-19 en México, publicado el 11 de enero del 2021, el cual es el documento rector para esta Política de Vacunación, que tiene como objetivo la disminución de la carga de la enfermedad y defunciones causadas por la COVID-19, mediante la inmunización de cuando menos $70 \%$ de la población y lograr la inmunidad de rebaño (100\% de personal de primera línea y 95\% de la población a partir de los 16 años cumplidos). ${ }^{8}$

A partir del 11 de diciembre del 2020, la Comisión Federal para la Protección contra Riesgos Sanitarios (COFEPRIS) otorgó autorización para uso de emergencia a la vacuna de Pfizer-BioNTech y, posteriormente, el 04 de enero del 2021 otorgó el mismo dictamen a la vacuna desarrollada por AstraZeneca y la Universidad de Oxford. ${ }^{9}$ El día 02 de febrero del 2021, después de aparecer los resultados de la fase III publicados en la revista The Lancet, donde se presentó que tiene una eficacia de $91.6 \%$, la COFEPRIS dio la autorización para su uso dentro de territorio mexicano. ${ }^{10}$

\section{GRUPOS Y PERSONAL DE PRIORIZACIÓN}

1. Personal sanitario que enfrenta COVID-19.

2. Personas adultas mayores.

3. Personas con comorbilidad o comorbilidades.

4. Personal docente de las entidades federativas en semáforo epidemiológico verde.

5. Resto de la población (mayor a 16 años).

Se desarrolló en una primera etapa que funcionara a su vez como calibración en la Ciudad de México y Coahuila. Posteriormente, se incorporó en la primera etapa a los estados de Querétaro, Estado de México y Nuevo León. A partir del día 12 de enero del año 2021 se amplió la estrategia al resto del país, de acuerdo con lo establecido inmunizando al personal de atención directa a pacientes COVID-19 en las 14 mil unidades hospitalarias que constituyen la Red IRAG (red de hospitales Infección Respiratoria Aguda Grave).

\section{La logística del proceso de vacunación es el siguiente:}

1. Ingreso y confirmación de turno (previo registro llevado a cabo en instituciones de salud o en el caso de la población abierta por la Secretaría del Bienestar).

2. Filtro sanitario y triaje respiratorio.

3. Mesa de registro.

4. Mesa de vacunación. 
5. Observación de 30 minutos (si presenta algún efecto adverso de la vacuna, brindar atención inmediata).

6. Salida.

Para la población abierta se estableció la estrategia federal denominada «Operación Correcaminos», cuyo objetivo es lograr la cobertura de manera eficaz y eficiente, teniendo la coordinación general el presidente Andrés Manuel López Obrador y los 32 subcoordinadores estatales. Se consideran 10 mil puntos de vacunación en los 32 estados de la República Mexicana con brigadas de 12 miembros (personal de salud, SEDENA, Marina, servidores de la nación adscritos a la Secretaría del Bienestar y personal voluntario de ser necesario). Las fechas de vacunación, grupo a vacunar y cantidad de vacunas a aplicar se pueden revisar en la Tabla 3.

A finales de enero del 2021 se ha vacunado a 19,734 docentes de instituciones públicas y privadas en todos los niveles de Campeche (único estado en semáforo verde desde el mes de noviembre del 2020).

La vacunación para personas adultas mayores se realizará con enfoque en las zonas rurales consideradas como vulnerables territorialmente por su lejanía y dispersión; progresivamente irán avanzando las brigadas hasta llegar a las áreas metropolitanas. Se ha desarrollado para el registro de vacunación de adultos mayores la página: https:// mivacuna.salud.gob.mx/index.php en la cual deberán realizar su registro para ser considerados en el proceso de vacunación. Aquellos que no puedan registrarse por alguna razón, las Brigadas correcaminos serán los encargados de atenderlos directamente.

Para el seguimiento y vigilancia epidemiológica se implementó el Sistema de Vigilancia de los ESAVI (Eventos Supuestamente Atribuidos a la Vacunación o Inmunización) para poder identificar de manera temprana y monitorizar los eventos adversos relacionados con la aplicación de la vacuna, así como dar respuesta rápida que permita minimizar el impacto negativo en la salud debido a la aplicación, componentes y proceso de administración de la vacuna. ${ }^{11}$

\section{CONCLUSIONES}

El proceso de vacunación en México es lento como en todos los países del mundo, ya que la oferta es poca en comparación a la demanda a nivel mundial, se hizo un gran esfuerzo por parte de la comunidad científica y de los laboratorios por tener lista una vacuna en un tiempo mínimo (menos de un año), pero las necesidades de tener un reparto equitativo y lineamientos adecuados para la distribución dentro de los países han sido un obstáculo adicional que en algunos países ha sido superado con éxito a partir de adquirir una gran cantidad de vacunas y sus sistemas de salud fortalecidos con capacidad de distribución, mientras que en países como México la logística y adquisición han estado en la opacidad y/o con datos parciales de cómo será la vacunación, uno de los momentos anecdóticos de controversia fue la aprobación de la vacuna Sputnik V, en donde de manera «informal»se reunió el día 14 de enero del 2021 el Comité de Moléculas Nuevas, que es el Comité de COFEPRIS, encargado de evaluar y dictaminar el uso de medicamentos o vacunas y el día 02 de febrero del presente año, la empresa Laboratorios de Biológicos y Reactivos de México S.A. de C.V. (Birmex) para solicitar registro ante la COFEPRIS para el uso de emergencia de la vacuna Sputnik V. ${ }^{12,13}$ Otro de los puntos importantes que deberá realizar el gobierno mexicano es cumplir con lo presentado en el documento oficial de vacunación, el cual hasta el momento se ha cumplido parcialmente, ya que en el orden que aparece, los docentes estaban después del personal de salud de primera línea, los adultos

Tabla 3: Etapas de la estrategia de vacunación en México.

\begin{tabular}{cccr} 
Etapa & Fecha & Grupo a vacunar & Cantidad (millones) \\
\hline Primera & Diciembre 2020 a febrero 2021 & Personal de salud de primera línea de control de la COVID-19 & 1.1 \\
Segunda & Febrero a abril 2021 & Personal de salud restante y personas de 60 y más años & 14.4 \\
Tercera & Abril a mayo 2021 & Personas de 50 a 59 años & 12.7 \\
Cuarta & Mayo a junio 2021 & Personas de 40 a 49 años & 16.2 \\
Quinta & Junio 2021 a marzo 2022 & Resto de la población & 49.2 \\
\hline
\end{tabular}

Fuente: Secretaría de Salud (2020). 
mayores y personas con comorbilidades, sucesivamente. En este momento en el estado de Campeche, uno de los estados que permaneció tres meses en color verde según el semáforo epidemiológico, y que actualmente está en color amarillo, fueron vacunaron los docentes a pesar de estar en el cuarto lugar de vacunación.

\section{REFERENCIAS}

1. De Clercq E. Antivirals and antiviral strategies. Nat Rev Microbiol. 2004; 2 (9): 704-720.

2. Dong L, Hu S, Gao J. Discovering drugs to treat coronavirus disease 2019 (COVID-19). Drug Discov Ther. 2020; 14 (1): 58-60.

3. Mishra SK, Tripathi T. One year update on the COVID-19 pandemic: Where are we now? Acta Trop. 2021; 214: 105778.

4. Lurie N, Sharfstein JM, Goodman JL. The development of COVID-19 vaccines: safeguards needed. JAMA. 2020; 324 (5): 439-440. doi: 10.1001/jama.2020.12461.

5. Singhal T. A review of coronavirus disease-2019 (COVID-19). Indian J Pediatr. 2020; 87 (4): 281-286.

6. Cao B, Wang Y, Wen D, Liu W, Wang J, Fan G et al. A Trial of lopinavir-ritonavir in adults hospitalized with severe Covid-19. N Engl J Med. 2020; 382 (19): 1787-1799.

7. Chen C, Zhang Y, Huang J, Yin P, Cheng Z, Wu J et al. Favipiravir versus arbidol for COVID-19: a randomized clinical trial. Med Rxiv. 2020; 2020.2003.2017.20037432.

8. Gobierno de México. Política Nacional de Vacunación contra el virus SARS-CoV-2, para la prevención de la COVID-19 en México. Documento Rector. Disponible en: https://coronavirus.gob.mx/ wp-content/uploads/2021/01/PolVx_COVID_-11Ene2021.pdf

9. Comisión Federal Para la Protección Contra Riesgos Sanitarios (COFEPRIS). Registro sanitario para uso de emergencia de producto con denominación distintiva Pfizer-BioNTech COVID-19 VACCINE. 11 de diciembre de 2020.

10. Logunov DY, Dolzhikova IV, Shcheblyakov DV, Tukhvatulin AI, Zubkova OV, Dzharullaeva AS et al. Safety and efficacy of an rAd26 and rAd5 vector-based heterologous prime-boost COVID-19 vaccine: an interim analysis of a randomised controlled phase 3 trial in Russia. Lancet. 2021; 397 (10275): 671-681. doi: 10.1016/ S0140-6736(21)00234-8.

11. Dirección General de Epidemiología. Manual de procedimientos estandarizados para la vigilancia epidemiológica de eventos supuestamente atribuibles a la vacunación o inmunización (ESAVI). Disponible en: https://epidemiologia.salud.gob.mx/gobmx/salud/ documentos/manuales/42_Manual_ESAVI.pdf

12. Valadez B. Birmex pedirá registro de uso de emergencia para vacuna anticovid Sputnik V: Ssa. MILENIO. 01 de enero de 2021. Disponible en: https://www.milenio.com/politica/birmex-pediraregistro-emergencia-vacuna-sputnik

13. Moreno M. Hugo López-Gatell explica sobre vacuna rusa Sputnik V. Imagen Radio. 02 de febrero de 2021. Disponible en: https://www. imagenradio.com.mx/hugo-lopez-gatell-explica-sobre-vacuna-rusasputnik-v

Conflicto de intereses: Los autores declaran no tener conflicto de intereses.

Financiamiento: La presente investigación no ha recibido ninguna beca específica de agencias de los sectores públicos, comercial, o sin ánimo de lucro.

\author{
Correspondencia: \\ José Eduardo Orellana Centeno \\ E-mail: jeorellano@unsis.edu.mx
}

